\title{
A METHOD FOR THE ACCURATE MEASUREMENT OF SHORT-TIME INTERVALS.
}

\author{
By Harvey L. Curtis and Robert C. Duncan.
}

\begin{abstract}
.
A method is described whereby the time between two events which occur less than a second apart can be determined with a high degree of accuracy. The method consists in ruling on a moving film a uniform time scale simultaneously with the recording of the events to be studied. The time scale is obtained by throwing flashes of light on the film, the interval between flashes being governed by a special shutter fastened directly to and operated by the prongs of a tuning fork.

The shutter consists of two slotted, aluminum vanes mounted, respectively, on the two prongs of a tuning fork, the slots being parallel to the axis of the fork and so adjusted that when the tuning fork is at rest the vanes overlap and the slots coincide. As the fork vibrates the slots coincide twice during each vibration, and thus form a shutter whose frequency is twice that of the tuning fork. The shutter is brilliantly illuminated, and the light which passes through as a flash when the shutter is opened is focused on the film by means of a suitable optical system.

Very satisfactory results are obtained using a 500-cycle fork, driven by impulses from a roo-cycle fork. This divides the film in thousands of seconds and makes it possible to read time intervals directly from the film with the unaided eye with an error of not more than one ten-thousandth of a second. By using a comparator the error is reduced to a few millionths of a second.

The article includes a complete discussion of the necessary conditions for the best results, the sources of error inherent in the method, and the accuracy which may be expected.
\end{abstract}

\section{CONTENTS.}

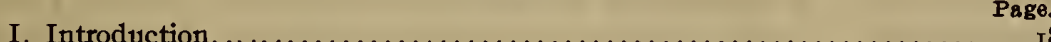

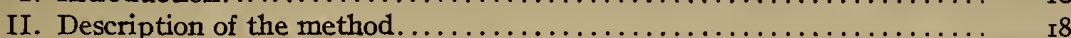

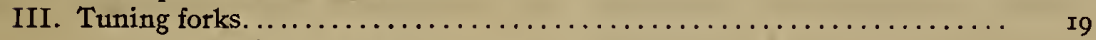

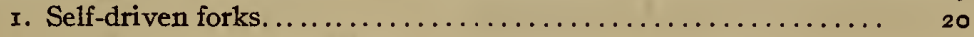

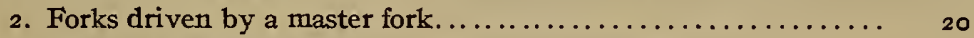

3. Freely vibrating forks. $\ldots \ldots \ldots \ldots \ldots \ldots \ldots \ldots \ldots \ldots \ldots \ldots \ldots, 22$

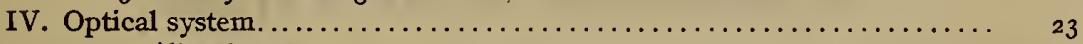

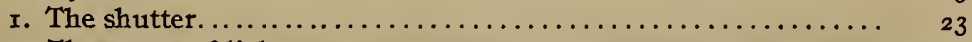

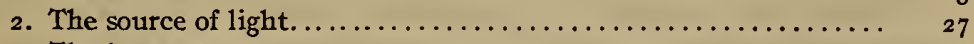

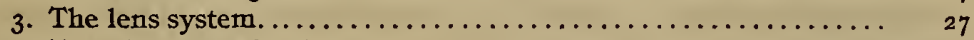

4. The photographic film............................ 28

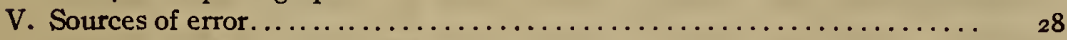

I. Errors in timing lines........................... 28

2. Error in measuring recorded interval.................. 30

3. Errors in recording mechanism............................ 3 I

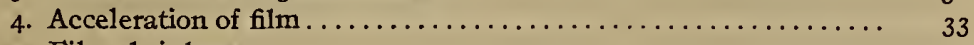

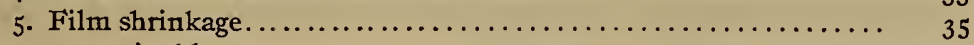

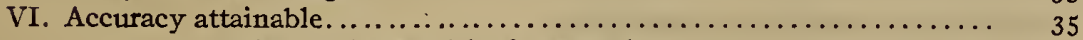

I. Intervals of a thousandth of a second..................... 36

2. Intervals of a hundredth of a second.................... ${ }_{36} 6$

3. Intervals of a tenth of a second $\ldots \ldots \ldots \ldots \ldots \ldots \ldots \ldots \ldots \ldots, \quad 36$

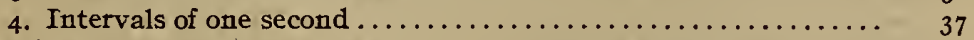

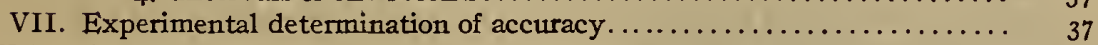

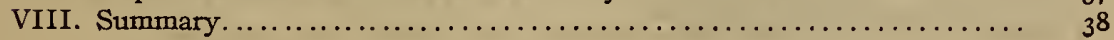




\section{INTRODUCTION.}

In December, 1916, the Bureau of Standards was requested to make measurements of certain time intervals in connection with the firing of large naval guns. A spark chronograph was first devised, which gave fair results. The work done with this showed the need of a method which had more flexibility and greater accuracy. The increased flexibility was readily obtained by the use of the oscillograph, by means of which events occurring at a distance can be recorded on a photographic film, but to obtain the necessary accuracy a method of measuring the speed of the film had to be developed. We first tried to record on the oscillograph film flashes from a mirror attached to the prong of a tuning fork. While this gave a satisfactory time record, the necessary apparatus was too bulky to serve our purpose. We next produced the flashes by a shutter operated by a small motor driven synchronously by a tuning fork. ${ }^{1}$ By means of this, very satisfactory timing lines were obtained on the films, but it was found that the hunting of the motor introduced appreciable errors. It was then decided to use a tuning fork ${ }^{2}$ as a shutter to interrupt the beam of light. With proper care this method will give results of the highest precision.

While this method was developed in connection with the oscillograph, yet it is applicable to any system which records photographically. In this article the general principles underlying the timing method are discussed and examples given to show the accuracy that can be attained.

\section{DESCRIPTION OF THE METHOD.}

The method consists in producing simultaneously on a moving photographic film a record of the occurrence of the events under consideration and timing lines from a tuning fork acting as a shutter. The essential feature of the method is the tuning-fork shutter, which produces a ruled time scale on the developed film. Several different methods have been used for recording the occurrence of the events. Of these the oscillograph can be adapted to the largest number of problems.

The shutter consists of two opaque vanes in which narrow slits have been cut and which are mounted on the prongs of a tuning

This is the phonic wheel of L,e Cour and Rayleigh. Our apparatus was largely copied from that of Dr. H. B. Williams, of Columbia University.

${ }^{2}$ First described by Lockler and Boltzman in Ann, d. Phys. u. Chem., 141, p. 321; 187o. 
fork. The vanes are so mounted that the slits are parallel to the axis of the fork, and the two slits coincide when the fork is at rest. When the fork vibrates, the two slits will coincide twice during each complete period of the fork. A picture of a fork with the vanes attached is shown in Figure 2.

In the use of the fork the slit is brilliantly illuminated and an optical system arranged to throw an image of the slit across the moving photographic film. Then successive images of the slit will appear on the developed film as lines perpendicular to the direction of motion of the film. These lines are called timing lines. In Figure $I$ is given a diagram of the tuning fork and

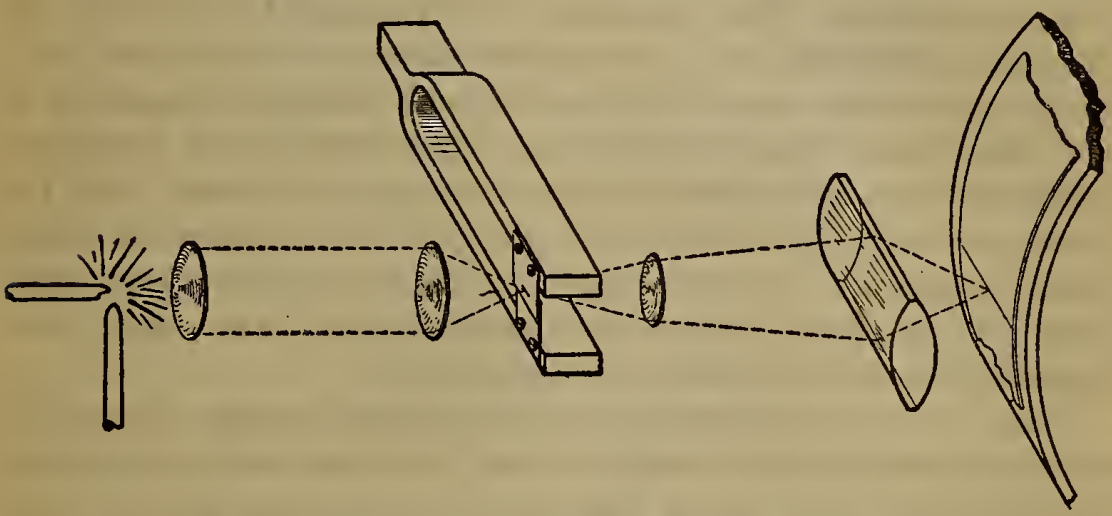

FiG. I.-Diagram of a tuning fork and optical system.

The opening produced by the two coincident slots in the vanes of the fork is brilliantly illuminated. A long, narrow image of this opening is thrown on the photographic film by means of one spherical and one cylindrical lens. With the fork vibrating and the film moving, lines will be produced on the film with $a$ definite time interval between them.

optical system as used in an oscillograph, while in Figure 3 is given a sample record. It will be noted that the timing lines extend only part way across the film. This is desirable where the event is one which can not readily be reproduced or where the highest accuracy is desired, since the timing lines sometimes obscure the record of an event. In general, it is desirable to have the lines extend across the film, so that the time between events can be read directly from the film without the use of a comparator.

\section{TUNING FORKS.}

The accuracy of the method will primarily depend upon the accuracy of the tuning forks. In discussing this accuracy it is convenient to classify tuning forks according to the method used 


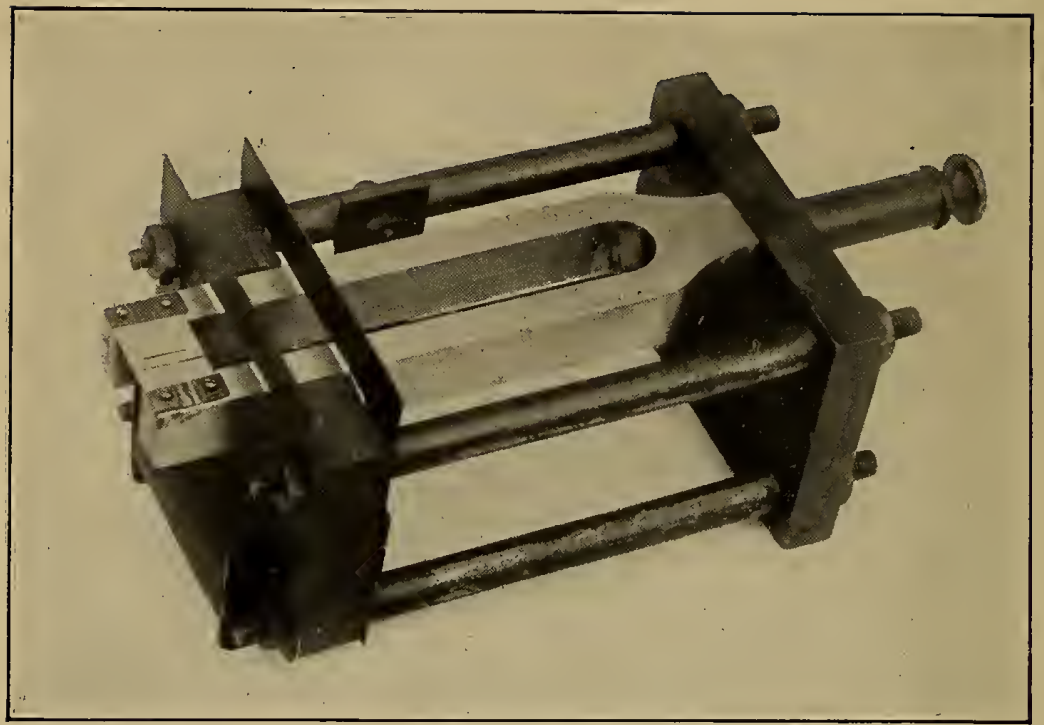

FIG. 2.-A 500-cycle tuning fork with shutter and mounting.

The shutter consists of two slotted vanes mounted on the two prongs of the fork. When the fork vibrates, the slots permit a flash of light to pass twice in each vibration. The mounting holds an electromagnet which is used in driving the fork.

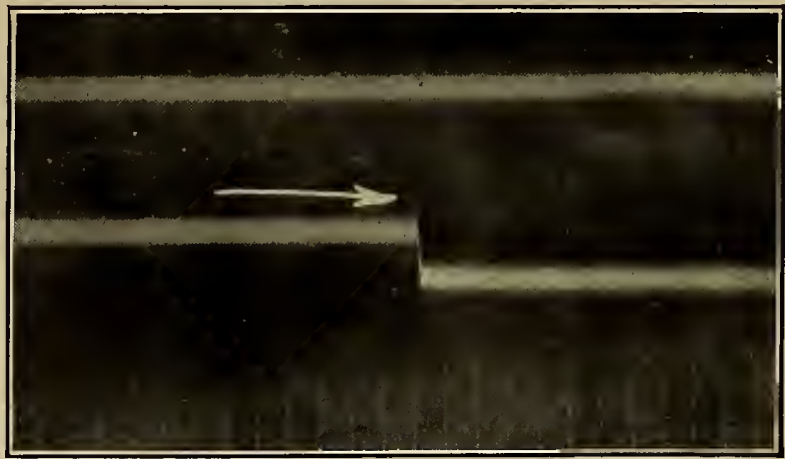

FIG. 3.-Characteristic record when tuning-fork shutter is used with an oscillograph.

The timing lines are shown below, the time between them being onethousandth of a second. The traces of two oscillograph elements are above, with a sharp break in one near the point of the arrow. 
in maintaining their vibrations. Three classes will be discussed: (I) Self-driven forks, (2) forks driven by a master fork, and (3) freely vibrating forks.

\section{SELF-DRIVEN FORKS.}

A self-driven fork is one in which the fork itself operates a device which applies a force to the prongs once during each vibration in such a manner as to maintain its vibrations. The most common device consists of an electromagnet and some arrangement whereby the vibration of the fork changes the current in this electromagnet. The electromagnet is so placed that, when energized, it exerts a force on the prongs of the fork in their direction of vibration. The variation in current may be produced by contacts on the fork, by a microphone placed on or near the fork, or by an electron tube which amplifies the current induced in a coil placed near the fork. The two latter methods are comparatively new and the authors have not seen any published data on the accuracy of forks with these types of drive. However, forks using contacts to vary the current have so long been used that they are generally called electrically driven forks, and their accuracy has been frequently investigated. ${ }^{3}$

With a well-constructed fork having balanced prongs, the period when electrically driven, will not vary by more than 0.2 per cent from its period when freely vibrating, so that an error greater than this could not be introduced by variations in the method of drive. Variations due to ordinary fluctuations of contacts, voltages, etc., will not affect the period of such a fork by more than o.r per cent. With sufficient care a much higher degree of accuracy can be obtained. Dadourian succeeded in maintaining a constancy of 2 parts in 100,000 for several hours. However, cases have arisen where variations in the driving constants will change the period by 5 per cent. Hence, it is necessary to investigate each fork.

\section{FORKS DRIVEN BY A MASTER FORK.}

It is entirely feasible to use electrically driven forks having a frequency as high as 250 vibrations per second. We have, however, experienced great difficulty in attempting to drive 500cycle forks directly, and have adopted a method of driving them with a master fork. ${ }^{4}$

\footnotetext{
${ }^{3}$ A very complete study of several forks and a comparison of the data obtained with that of previous investigations was made by Dadourian: Phys. Rev. (2), 13, p. 337; rgrg.

1 The method is similat to one described by Helmholtz; Die Lehre von den Tonempfindungen, p. 184 , 3d ed.; 1870.
} 
The master fork is electrically driven and carries an extra contact. This extra contact is connected in series with the magnet of the high-frequency fork and a suitable source of electric power. If the frequency of the high-frequency fork is an exact multiple of that of the low-frequency fork, there is little difficulty in obtaining vigorous vibrations. We have successfully driven 500 -cycle forks by a 50-cycle master fork. Less difficulty is experienced, however, when the ratio of periods is smaller. Very satisfactory results have been obtained using a roo-cycle fork to drive a 500-cycle one. In fact, as many as four 500cycle forks have been driven simultaneously by one master fork using but one extra contact.

Obviously, the accuracy attainable by this method is no greater than that of the self-driven master fork. However, for films moving at speeds above $500 \mathrm{~cm} / \mathrm{sec}$. the 500 -cycle fork produces a much more satisfactory record than would be produced by a Ioo-cycle or even a 200 -cycle fork. The timing lines are sharper and the time scale is divided into more convenient units. With a film on which there are timing lines from a 500-cycle fork it is possible to read a time interval in thousandths of a second directly and estimate to ten-thousandths, using only the unaided eye. In many problems greater accuracy is not needed.

However, for problems demanding the highest precision obtainable, the method is not satisfactory in that small periodic errors are introduced by the master-fork drive. These are caused by the fact that the frequency ratio of the forks can not be continuously maintained an exact integer. The driven fork receives an impulse, vibrates freely for several vibrations, then receives another impulse, etc., the process being continuously repeated. If the number of free vibrations of the driven fork between impulses is not an exact integer, it is forced into step with the master fork as each impulse is received. Hence, the period of those vibrations in which the fork is given impulses are either longer or shorter than the periods of the other vibrations. This produces a periodic error in the timing record, which will always be small, since the two forks must be very accurately timed in order to obtain an amplitude of the driven fork sufficiently large to give satisfactory timing lines. Careful comparator measurements are necessary to detect this periodic error, and it is generally inappreciable in comparison with the errors introduced by the electric drive of the master fork. 


\section{FREELY VIBRATING FORKS.}

The inaccuracies of the preceding method can be obviated by arranging to open the circuit which drives the high-frequency fork during the interval that the exposure is being made. Hence, during this interval the fork will be vibrating freely and will give the accuracy to be obtained from a freely vibrating fork.

The only important factors which affect a freely vibrating fork are its temperature, its amplitude of vibration, and its method of mounting. The temperature coefficient is approximately o.or per cent per degree centigrade, the frequency decreasing with increasing temperature. The results of a number of observers ${ }^{5}$ shows that this coefficient always lies between o.oro and o.or 4 per cent in the ordinary range of room temperature. Hence, if the temperature does not vary by more than $10^{\circ}$, the use of o.or per cent as the temperature coefficient will not introduce an error in the frequency of more than 0.04 per cent. For higher accuracy it is necessary to limit the temperature range or determine the temperature coefficient of each individual fork used.

Any effect of the fork mounting may be eliminated by calibrating it on the same base as that on which it is used. When this is not possible, careful consideration must be given to the method of mounting. With driven forks changes of o.I per cent have been observed, caused by changing a fork mounted on a heavy base from a solid pier to a shaky stool. "

An increase in the amplitude of vibration in a tuning fork increases its period. The maximum change recorded by Miller ${ }^{7}$ is less than I part in 10,000. However, the amplitudes which he used were small, whereas to produce satisfactory timing lines a fork must have a large amplitude. In order to obtain information concerning forks which have been used in this work, the following experiment was performed:

Two forks, Nos. 22 and 23 , were so arranged that they would have a double amplitude of vibration of about $\mathrm{I} \mathrm{mm}$ when driven by a master fork. The set-up was then arranged so that the relative frequency of the forks could be determined in the first o. I second after shutting off the electric drive, and also approximately one second later when the amplitude of fork No. 22 had

See Dadourian, loc. cit., for a list of observers and the values they obtained. Some of this data is on driven forks, but there seems to be no difference in the temperature coefficient of driven and freely vibrating forks.

6 Dadourian, loc. cit.

7 Miller, D. C., Phys. Rev. (2), 1, p. 497 ; 1918. 
decreased to about $0.5 \mathrm{~mm}$ and of fork No. 23 to about $0.3 \mathrm{~mm}$. If we assume that the frequency of fork No. 22 remained constant over the entire period at 500 vibrations per second, then the film measurements gave the following values for the frequency of fork No. 23 :

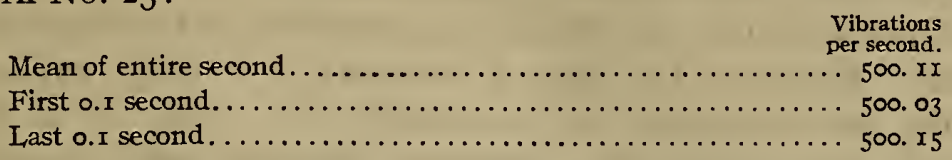

As a first approximation the increase in frequency is proportional to the decrease in amplitude. On this assumption the above data show that a change in amplitude of $0.1 \mathrm{~mm}$ causes the frequency to change by I part in Io,ooo. Hence, for the highest accuracy it is necessary to measure the amplitude of vibration and to apply corrections which must be experimentally determined.

\section{OPTICAL SYSTEM.}

While the accuracy of this method of measuring time intervals depends primarily upon the period of the tuning fork, yet the highest precision can be obtained only if a suitable optical system is employed. This consists of the shutter, the source of light, the lens system, and the photographic film.

\section{THE SHUTTER.}

The shutter, consisting of vanes on a vibrating fork, produces the flashes of light which make the timing lines on the moving photographic film. In order to make accurate measurements, these lines must be narrow and their edges sharp. The following discussion shows the conditions that must be fulfilled to obtain such lines.

The shutter is shown diagrammatically in Figure 4 . The vanes with their slits are moving in opposite directions, being attached to the prongs of the tuning fork. Since the fork is always near the middle of its swing during the time that the shutter is open, the velocity of the vanes is approximately uniform. Hence, on a stationary film there appears a line of light as the shutter begins to open, and the two edges move outward with uniform velocity until the shutter is completely open. At that time the edges of the shutter image instantly reverse their direction and move toward the center with the same velocity as they formerly moved outward. There is then a discontinuity in the velocity of the edges of the image at the time the shutter is completely open. 
If the film is moving with a velocity less than the velocity of the edge of the image, then no portion of the film is exposed during the opening and closing of the shutter which is not exposed when the shutter is at its maximum opening. In other words, the width of film exposed is independent of the velocity of the film, provided this velocity is less than that of the edges of the image. However, the time of exposure of a point within the timing line is affected by the velocity of the film. With a stationary film the exposure increases uniformly from the edge to center, so that the diagram which shows the exposure at each

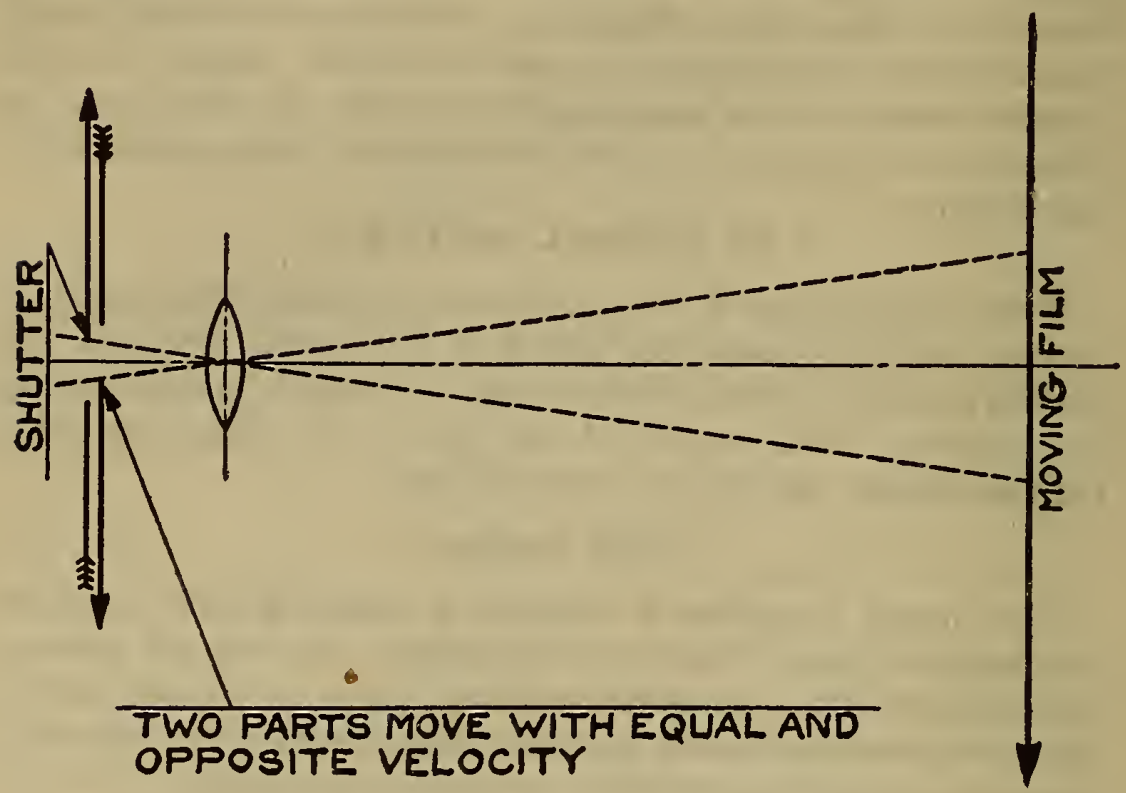

Fig. 4.-Diagram to show the operation of a tuning-fork shutter.

The width of a timing line depends not only on the width of the shutter opening and on the magnification of the lens system, but also on the velocities of the shutter vanes and of the photographic film.

point of a line drawn across the timing line is an isosceles triangle. In the case of a slowly moving film this diagram becomes an isosceles trapezium, the exposure being uniform over a band in the center of the timing line and decreasing linerally from this band to the edge. An increase in the velocity of the film decreases the exposure in the central portion and increases it toward the edges.

If the film is moving with a velocity greater than the velocity of the image of the edge of the shutter, then that point which is exposed at the instant the shutter begins to open is outside that 


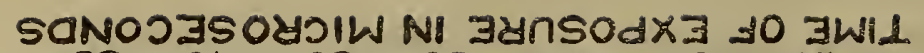
$001 \quad 06 \quad 08$ oL 09 os of of 02 of

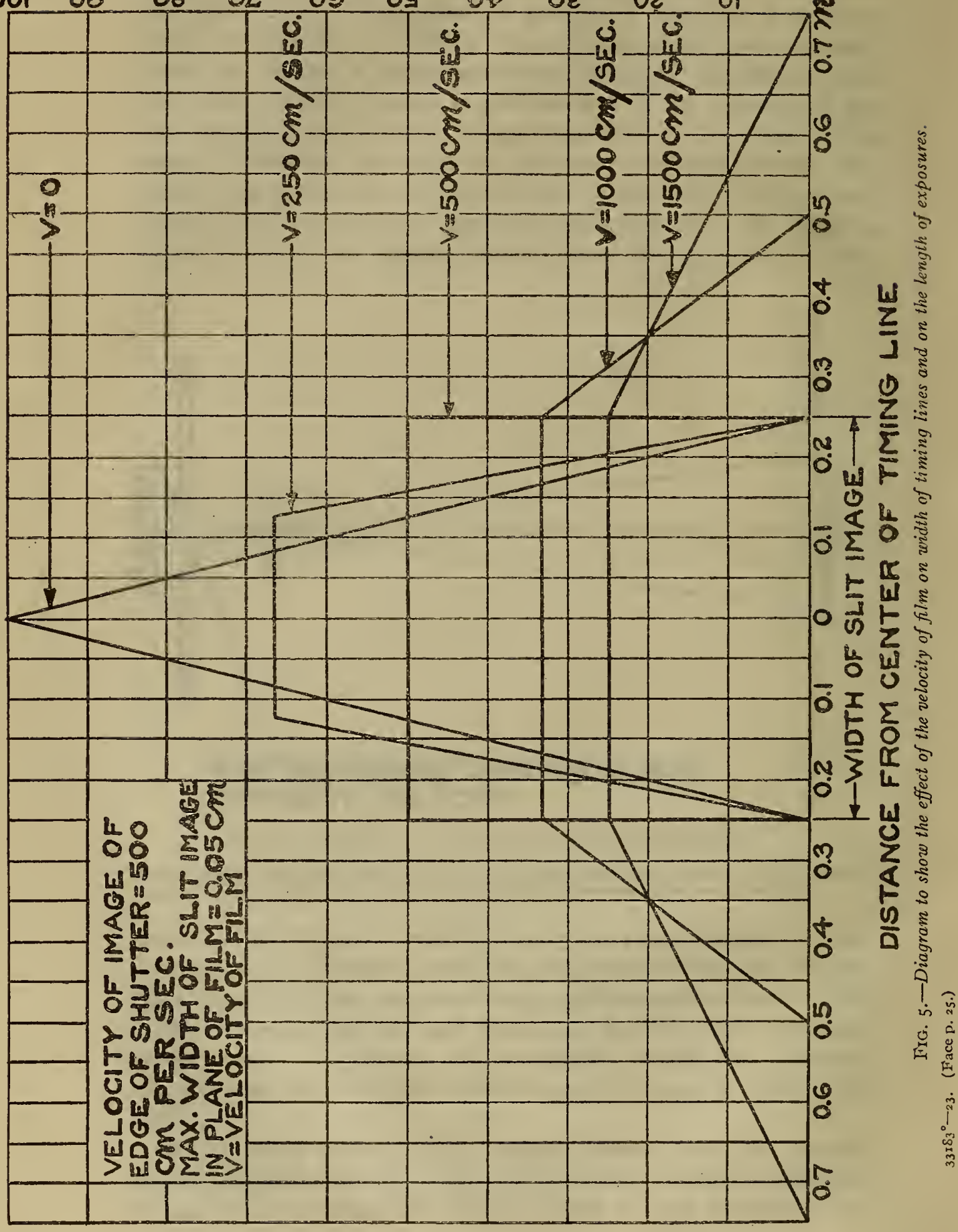


portion which is illuminated when the shutter is at its maximum opening. In this case the amount of film exposed does depend on its velocity, so that an increase of film velocity beyond the velocity of the image increases the width of the timing line and decreases the exposure in the center. However, the exposure diagram is still an isosceles trapezium, as in the case of the slowmoving film.

The width of exposure and the time of exposure for different velocities of the film are shown in Figure 5. The equations for constructing these diagrams were analytically determined by the methods of geometrical optics. This analysis shows that the exposure diagram in which the time of exposure is plotted as ordinates with distance along the film as abscissa is always an isosceles trapezium, one of whose bases is the width of exposure on a stationary film. The height of this trapezium is $\frac{S}{V+v}$, where $S$ is the width of exposure on a stationary film, $V$ the velocity of the film, and $v$ the velocity of the image of an edge of the shutter slit. This height gives the time of maximum exposure. The length of the bases of the trapezium is given in Table 1 . The length of the shorter base shows the length of film which receives the maximum exposure, while the length of the longer base shows the total amount of film exposed, thus determining the width of the timing line.

TABLE 1.-Length of Bases of Exposure Trapezium.

\begin{tabular}{|c|c|c|}
\hline Velocities, film image. & Length oi shorter base. & Length of longer base. \\
\hline$V<v$ & $\frac{S v}{v}$ & $S$ \\
$V=v$ & $S$ & $S S$ \\
$V>v$ & $S$ & $\frac{S V}{v}$ \\
\hline
\end{tabular}

The time of exposure and the width of exposure depend solely upon the velocities of the film and of the edges of the image and upon the width of exposure when film is stationary. The velocity of the edge of the image in its turn depends upon the velocity of the prongs of the tuning fork and upon the magnification of the optical system, while the width of exposure on a stationary film depends upon the width of the slits in the vanes of the shutter and upon the magnification of the optical system. 
Let

$K=$ magnification of optical system in the direction of the width of the slits.

$W=$ width of slit on each vane.

\section{Then}

$S=K w=$ width of exposure on a stationary film.

Also let

$a=$ amplitude of vibration of fork.

$n=$ frequency of fork.

$v=2 \pi a n k=$ velocity of image of edge of shutter.

By using these values of $S$ and $v$ all constants of the timing lines are obtained in terms of the constants of the fork and the velocity of the film.

In order to have narrow timing lines, it is necessary that the slits in the tuning-fork vanes shall be narrow and that the magnification shall be small. Also, the velocity of the image must be equal to or greater than the velocity of the film. To obtain a large velocity of image with small magnification, it is necessary that the amplitude of vibration of the fork shall be large and its frequency high. This explains why it has been necessary to use 500-cycle forks to get satisfactory timing lines on high-speed films.

The sharpest timing lines will be obtained when the ratio of the velocity of the image to the velocity of the film is unity. In this case the exposure is uniform over the timing lines, and the edges are as sharp as the photographic processes will produce. As this ratio becomes either greater or less than unity, there is a shading off of the exposure at the edges, which makes the edges of the timing lines less sharp.

In Figure 6 is a reproduction of a film on which are three sets of timing lines. In taking these the velocity of the film was varied while the amplitude of the fork, and hence the velocity of the image of the edge of the slit, remained constant. In the middle set of timing lines the two velocities are very nearly the same, the film having a velocity of $320 \mathrm{~cm} / \mathrm{sec}$. and the image $340 \mathrm{~cm} / \mathrm{sec}$. the broad and rather indistinct timing lines were made with a film speed of $I, 050 \mathrm{~cm} / \mathrm{sec}$, while the slowest speed was $53 \mathrm{~cm} / \mathrm{sec}$.

The middle set of timing lines has the sharpest and most distinct edges, though the width of these lines is the same as those made at a much slower speed. The timing lines made with a 
high velocity of film are broad and have an indistinct edge. Within experimental error their width is that which would be computed from the preceding table.

\section{THE SOURCE OF LIGHT.}

As the time of exposure of the film in the center of a timing line depends upon the period of the fork, a more brilliant source of light is required for forks of high frequency than for forks of low frequency. An incandescent lamp has been used to get timing lines with forks having a frequency as high as 250 cycles per second. For 500-cycle forks an arc lamp is the most satisfactory source of light.

Some experiments have shown that it is possible to use a tungsten wire in air heated to a very high temperature by an electric current. This wire can be placed directly behind the slit, so that no condensing system is required. By means of relays a very heavy current is thrown on this wire just before the shutter opens, which exposes the film. The wire is heated very nearly to its melting point, and while it oxidizes rapidly it will last for the tenth of a second or more that is required. This method has the disadvantage that it produces a cloud of tungsten oxide which settles on the surrounding objects. It is possible that this would be obviated by using a metalized carbon filament.

\section{THE LENS SYSTEM.}

Since ordinarily the source of light must be some distance from the tuning fork, some condensing system is necessary to illuminate the slit with sufficient brilliancy. Two simple spherical lenses have been found satisfactory, one placed near the source of light to produce a parallel beam and the other near the tuning fork to concentrate the light on the slit.

The important part of the lens system is that part which produces an image of the slit on the photographic film. If the slit is near the film, a single lens may suffice; but if the distance between the two is more than 30 or $40 \mathrm{~cm}$, two lenses will be required, one to bring the light into a parallel beam and the other to focus this beam on the film. If long timing lines are desired, this second lens should be a short focus cylindrical lens. Such a lens will produce narrow timing lines but permit them to extend the full width of the film.

The aberrations in a lens system always cause a slight blurring of the image. The effect of these aberrations in the system 
under consideration is to increase the width of the timing lines over that determined by geometric optics and to make their edges less sharp. However, by keeping the angular opening of the lenses small the effect of the aberrations is inappreciable.

\section{THE PHOTOGRAPHIC FILM.}

When the speed of the recording film is high; that is, I,, 00 to $I, 500 \mathrm{~cm} / \mathrm{sec}$., it is necessary to have a very sensitive film in order to obtain satisfactory results. As the sensitiveness decreases with age, it is important to have fresh films, which should be developed to show as great a contrast as possible. Tropical developer is very useful when the temperature of the developing solutions can not be controlled.

\section{SOURCES OF ERROR.}

There are a number of factors other than the tuning fork which may introduce errors. The most important of these are: (I) Errors in measuring distance between timing lines, (2) errors in measuring the distance on the film which corresponds to the desired time interval, (3) inaccuracies in the adjustment of the recording mechanism, (4) acceleration of the film during the recording, and (5) unequal expansion or contraction of film during the developing and drying process.

\section{ERRORS IN TIMING LINES.}

Since the time between the two events is obtained by comparing the distance recorded on the film with the distance between timing lines, an error in the position of the timing lines on the film or in measuring this position will introduce an error in the measured time. To avoid errors in the position of the timing lines, it is necessary that the shutter slits shall be so adjusted that the longitudinal center lines of the two slits shall coincide when the fork is at rest. When the adjustment is imperfect, the center lines of the slits may be parallel but not coincident, or they may lack parallelism but coincide at one point.

In the first case the timing lines will be parallel, but the length of each alternate timing interval is different from the intervening ones. In the second case alternate timing lines will be parallel, but the intervening ones will be at an angle. In this case a line may be drawn along the film which will be cut at equal intervals by the timing lines. The point on the timing lines which fall on this line are the images of that point at which the longitudinal 
Scientific Papers of the Bureau of Standards, Vol. 19.

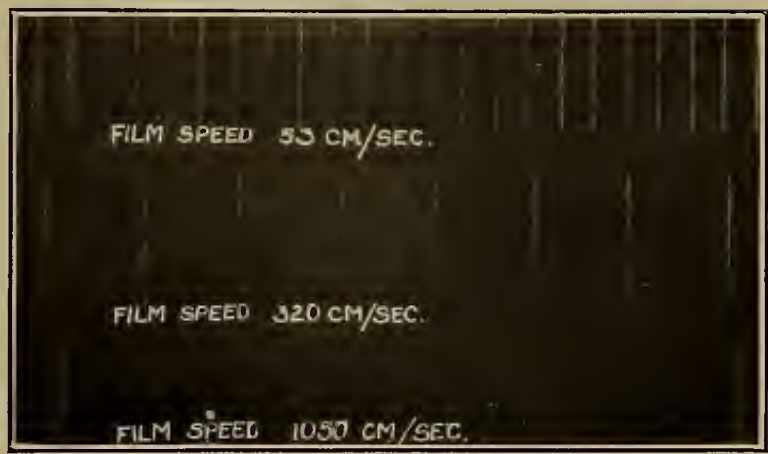

FIG. 6.-Record showing width of timing lines with three different velocities of films.

The same fork was used to make all three of these records. If the film speed is too high for a given fork, the timing lines are broad and indistinst, as shown in the lower part of the figure.

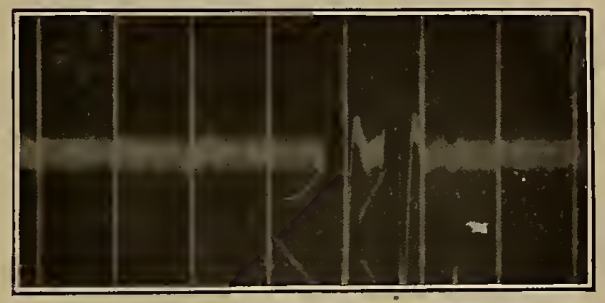

FIG. 7.-Record used to determine accuracy of different observers in reading film distances.

Over $I, 000$ measurements were made on this film by means of a comparator to see how well different observers arreed as to the distance between the breaks indicated by the arrows. (See p. 3r.) 
Scientific Papars of the Bureau of Standards, Vol. 19.

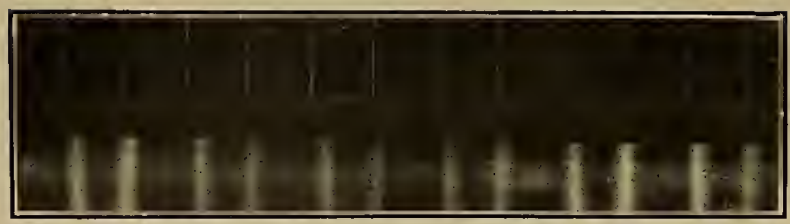

FIG. 8.-Records showing the effect of the amplitude of vibration on the character of timing lines.

In the upper record the fork was vibrating with a large amplitude, while in the lower record it had nearly stopped vibrating.

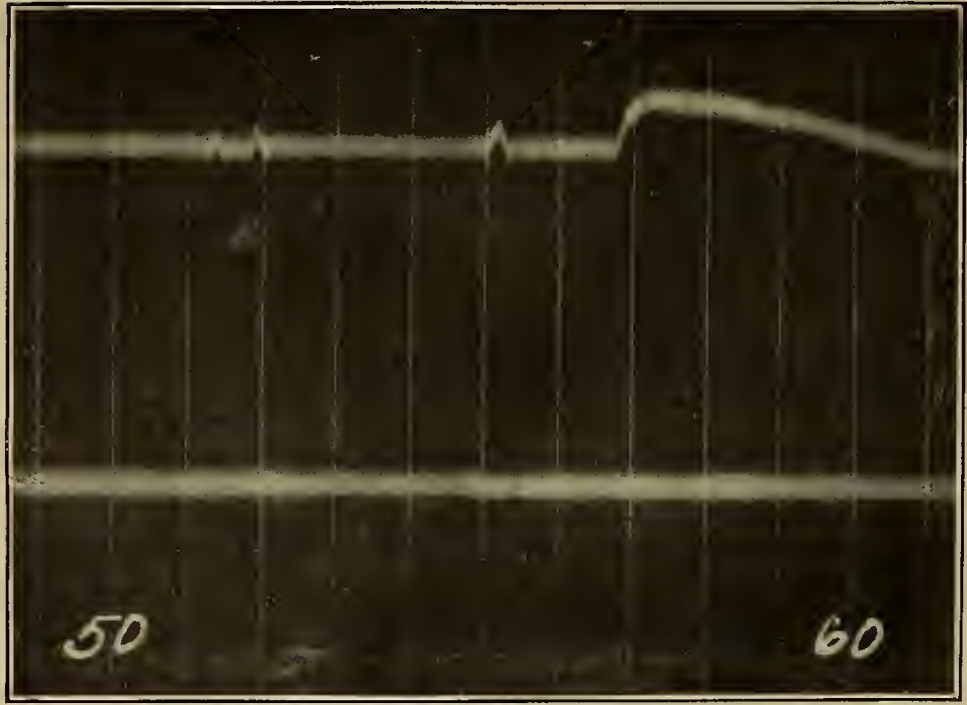

FIG. 9.-Record used to measure nonparallelism of timing lines.

These timing lines appear parallel, but careful measurements show that they are not. The results of these measurements are shown in Figure 9. 
center lines of the slits coincide when the tuning fork is at rest. If the point at which these center lines coincide does not fall within that part of the slits used to produce the timing line, the timing lines will not be equidistant at any point in their length.

The magnitude of the displacement of the timing lines for a given parallel adjustment of the slits depends upon the amplitude of vibration of the fork, being most noticeable when the amplitude is small. In Figure 8 is shown a record where the adjustment is not perfect and where the amplitude of vibration of the fork varies through a considerable range. When the amplitude is large, the timing lines are almost equally spaced, but with small vibrations each alternate timing line is considerably displaced.

Lack of parallelism of the slits does not often cause noticeable

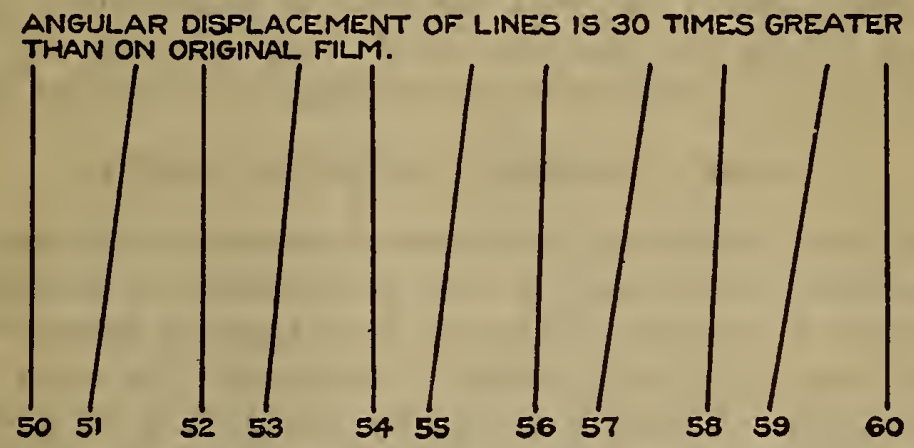

Frg. I0.-Diagram showing nonparallelism of lines of Figure 9 .

On the film the timing lines appear parallel but careful measurements gave the results recorded above.

errors in the timing lines. However, they can frequently be detected by careful measurements. Figure 9 is a reproduction of a part of a record which shows very excellent timing lines. However, measurements made at the top and bottom of the timing lines show a measurable lack of parallelism. This is greatly exaggerated in Figure ro. The line of equal spacing between timing lines falls on the film, while at both top and bottom timing intervals are alternately i per cent greater and I per cent less than the mean value.

While it is exceedingly difficult to obtain a perfect adjustment of the slits, yet they can be readily adjusted so that the difference between timing intervals does not vary by more than 5 per cent in the normal operation of the fork, while with care in adjustment the difference may be as small as 2 per cent. In such a case no error is introduced when the timing lines are used to read the 
time directly without the use of a comparator. However, when accurate measurements are to be made, it is necessary to measure an even number of timing lines and to measure them along a line drawn in the direction of the motion of the film.

The errors in measuring the position of the timing lines may depend to some extent upon the kind of measuring iristrument used, but are much more affected by the character of the timing lines themselves. If the measurements are made with a comparator using a micrometer microscope, the instrumental errors are negligible, but there will be an uncertainty in the setting of the cross hair of the microscope on the center of the timing lines. With sharp, narrow timing lines this error will not exceed $0.05 \mathrm{~mm}$ and will be very much less than this with good observers. Hence, with timing lines $\mathrm{I} \mathrm{cm}$ apart, the error in determining a timing interval will be less than one-half of $\mathrm{I}$ per cent, and with good observers may be reduced to approximately one-tenth per cent.

\section{ERROR IN MEASURING RECORDED INTERVAL.}

The time at which any event occurs is recorded on the film by a spot of light which is made to move perpendicular to the direction of motion of the film. This spot of light usually comes from a mirror which is given a motion at the instant the event to be timed occurs. A method frequently employed is to have the event close an electric circuit. This circuit produces a current through an element on the oscillograph (a high-frequency galvanometer which records photographically) which gives a sudden deflection to the beam of light.

When the recording mirror is stationary, the beam of light produces a straight trace on the film. When this beam is deflected, the trace will move at an angle from the original trace, the sharpness of this angle depending upon the speed at which the mirror moves. The most desirable condition for reading the time of this event accurately is to have this angle as near $90^{\circ}$ as possible. Hence, in using the oscillograph for timing purposes it is desirable that the event shall send a very large current through the galvanometer and also desirable that the element shall be damped as little as possible, so that it will move rapidly. It is only necessary that there be sufficient damping so that the element will reach its zero position before the occurrence of the next event which is to be recorded by that same spot of light. 
In order to determine the accuracy which can be obtained in reading the recorded interval between two events, a series of measurements was made of the distance between two events by Io different observers. Figure 7 is a reproduction of the film used for these measurements.

To determine the distance between the events, each observer made a set of 40 observations, taking alternate readings of the two events. Observations were made under three conditions, five of the observers taking a set of readings under all three conditions. The mean value and the probable error were then computed for each set of observations and for the entire group.

The results of the 1,000 observations are shown graphically in Figure II. It will be noticed that with the poorest observer under the most unfavorable conditions the error in determining the distance was not as great as $0.02 \mathrm{~mm}$. With the best observers the error is less than $0.005 \mathrm{~mm}$. Hence, if the distance on the film between the recorded events is as great as I cm, it will be comparatively simple to obtain an accuracy of one-fifth per cent, while with selected observers an accuracy of one-twentieth per cent may be obtained.

\section{ERRORS IN RECORDING MECHANISM.}

In order to determine accurately the time interval between two events, it is important that both events shall be recorded on the film by the same spot of light, and that the time lag between the occurrence of the event and the motion of the spot of light shall be the same for both events. The necessity for a single spot of light arises from the difficulty of seting up two optical systems which will have the same characteristics. For example, it is very difficult to so adjust the spots of light from two different elements of the oscillograph that they simultaneously record on a line which is perpendicular to the direction of motion of the film. This is essential if they are to give identical indications on the film of the time at which events occur.

The following example shows one method which has been used for recording two events by one spot of light. It was desired to measure the time which elapsed while a projectile was leaving the muzzle of the gun. An insulated bar was placed across the muzzle so that when the nose of the projectile left the gun it made contact with this bar. Likewise, an insulated steel finger was mounted on the muzzle so that it did not make contact with 


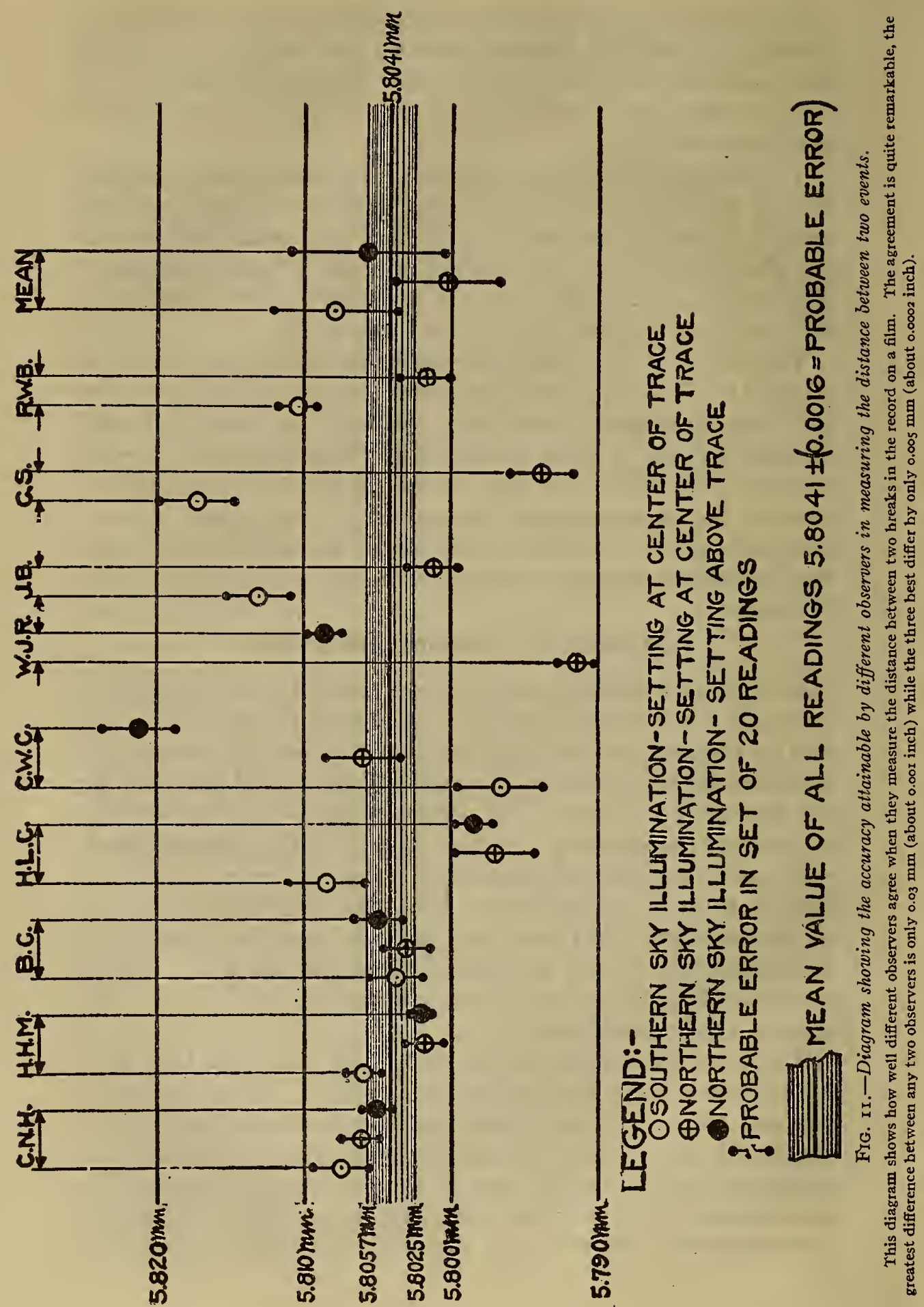


the shell proper, but did make contact as the rotating band left the muzzle. A circuit was then arranged to run from the gun, through an oscillograph element and a suitable battery, to the insulated bar and finger connected in parallel. As the projectile reached the muzzle the nose of the projectile made contact with the bar, producing a sudden deflection of the oscillograph element. However, this bar was immediately carried away by the projectile, thus again opening the circuit, which was closed again when the rifling band made contact with the finger. The parts of the recording mechanism are exactly identical, the only possible difference being the two contacts which are made on the projectile. As these are made at high speed, there can be very little difference between the two. In a time difference of o.oor second an accuracy of one-half of I per cent can be attained. However, if the circuits are altered and the time of contact of the bar is recorded on one oscillograph element and that of the finger on another oscillograph element the accuracy is considerably reduced.

Where it is not feasible to record the two desired events by the same spot of light, it is often possible to introduce a third event which will be recorded by each of the two spots of light which record the events under observation. The time difference between the auxiliary event and each of the two desired events is then determined in the manner described above, the desired time being the difference between these observed intervals.

As an example of the above, it was desired to determine whether the rotating band on the opposite sides of a projectile left the muzzle at the same instant; in other words, to see if the projectile had any yaw as it left the muzzle of the gun. Two fingers were fitted on opposite sides of the muzzle, so that they would make contact on the rotating band, but they could not be connected to the same oscillograph element, since the time interval was so short that the oscillograph element would not return to zero between the time of making the first and second contact. However, by using two oscillograph elements and connecting one to one rotating band finger and the other to the other finger while connecting both to the same muzzle bar, which is described above, the time difference in each case could be determined, and hence the time that one contact preceded the other.

\section{ACCELERATION OF FIIM.}

The method as outlined supposes that the film is moving with uniform velocity. This can never be accurately accomplished for 
several reasons. First, while the power supplied by the motor is, on the average, just equal to the friction in the moving mechanism, yet the friction will vary slightly from instant to instant, causing the motor to change its speed to compensate the change in power required. Hence, changes in friction will cause changes in the velocity of the drum. Second, variations in the voltage applied to the motor will cause variations in the speed of the motor. This can be largely obviated by the use of an independent storage battery to drive the motor, but this is not always feasible. Third, it is always necessary to throw some additional load on the motor at the instant that the shutter is opened in order to actuate a mechanism which will close the shutter at the end of a revolution. The inertia is quickly compensated, but the added frictional load will decrease the speed of the film for an appreciable length of time.

The obvious method of securing a sufficiently uniform motion of the film is to make the drum with a very large moment of inertia. Then, when there are sudden changes, either in the friction of the drum mechanism, the power of the driving motor, or the power consumed in the auxiliary apparatus, only slight changes in the velocity of the drum will be produced.

In our work we have used large drums which take a $150-\mathrm{cm}$ (6o-inch) film. These have a mass of about $5 \mathrm{~kg}$ and a moment of inertia of $2.3 \times 10^{6} \mathrm{~g} \cdot \mathrm{cm}^{2}$. Such a drum running freely in its bearings showed a deceleration of $170 \mathrm{~cm} / \overline{\mathrm{sec}}^{2}$ at a velocity of $190 \mathrm{~cm} / \mathrm{sec}$. When driven by a motor, the acceleration (positive or negative) did not exceed $100 \mathrm{~cm} / \mathrm{sec}^{2}$ Hence, in $\mathrm{I} / \mathrm{roO} \mathrm{sec}$. the velocity may change by $\mathrm{I} \mathrm{cm} / \mathrm{sec}$.

By properly choosing the timing lines to be measured the effect of a small acceleration of the film on the measured time can be made negligibly small. If the recorded interval is short, then the middle one of the measured timing lines should be near the center of the recorded distance. If the recorded interval is long, so that two sets of timing lines will be measured, then the middle timing line of the first set should be near the beginning of the recorded interval, and the middle one of the second set should be at the end of the recorded interval. With these precautions the error caused by acceleration of the film will be much less than other errors which can not be so readily eliminated. 


\section{FILM SHRINKAGE.}

In order to test the amount of film shrinkage, films were exposed in contact with a mirror through the silver surface of which lines $5 \mathrm{~cm}$ apart had been ruled. The films were then developed, and, when dry, distances between two different lines of the film were measured on the comparator and compared with the distance between these same lines on the original glass plate.

All of the films showed a shrinkage which varied from one-half to I per cent, depending on the kind of film and the method of development. For a given film with a definite method of development the maximum observed difference was 0.6 of I per cent. The shrinkage was so local and irregular that no method of correcting for it has been devised.

If the time interval is measured over the same portion of the film as is used for recording the events, no error is introduced in the results by unequal shrinkage of the film. While this condition can only be approached in practice, yet the error due to film shrinkage can be made small.

\section{ACCURACY ATTAINABLE.}

In determining the accuracy which can be attained in measuring short-time intervals by this method, three factors need to be considered, viz: (I) The mechanism which is used to record on the film the time interval under discussion, (2) the rate of the tuning fork, and (3) the determination of the time interval recorded on the film. The relative importance of these factors will depend upon the speed of film and upon the length of interval to be measured, while the precautions that must be observed in doing the experimental work will also depend upon the accuracy desired.

For very short-time intervals it is desirable to have a high velocity of film. However, mechanical as well as photographic difficulties arise when the speed is too high. While satisfactory records have been made with film speeds as high as $30 \mathrm{~m} / \mathrm{sec}$, yet it is seldom feasible to use speeds of more than $10 \mathrm{~m} / \mathrm{sec}$. The following discussion of the accuracy attainable for various time intervals assumes this film speed.

The accuracy with which the recorded time interval can be determined depends not only on the accuracy with which the recorded interval and timing intervals can be measured, but also on how completely the effect of film shrinkage and film acceleration can 
be eliminated. In the following discussion it is assumed that the error due to shrinkage and acceleration is no greater than the error due to measurements.

\section{INTERVALS OF A THOUSANDTH OF A SECOND.}

In measuring time intervals of the order of one-thousandth of a second the accuracy is generally limited by the recording mechanism. To obtain an accuracy of one-tenth per cent, it is necessary that the time error in the mechanism does not exceed one-millionth of a second. The difficulty of designing any mechanical device which will work with this precision can readily be appreciated when it is observed that the highest velocity projectiles move only a few hundredths of an inch in a millionth of a second. Only in special cases is it possible to design a mechanism which will give an accuracy better than i per cent.

The film measurements can be made with an accuracy of one or two tenths per cent on a good film. Hence, allowing for the errors due to film shrinkage, the recorded time interval can be determined with an accuracy of one-half per cent, or under favorable conditions of one-quarter per cent. The tuning fork will never be a source of error, since even an electrically driven fork can be depended upon to one-tenth per cent.

\section{INTERVALS OF A HUNDREDTH OF A SECOND.}

In determining the value of time intervals of the order of onehundredth of a second, the recorded time interval can be determined with an accuracy of one-tenth of I per cent. However, to obtain such an accuracy it is necessary that the rate of the tuning fork be carefully determined, since changes in methods of driving, temperature, etc., may cause variations in the rate by as much as one-tenth of I per cent. Also, the recording mechanism must be carefully designed in order to obtain an accuracy of onetenth per cent.

\section{INTERVALS OF A TENTH OF A SECOND.}

For time intervals of one-tenth of a second the recorded interval can be determined with an accuracy of one-hundredth of I per cent. If the recording apparatus is properly designed, then the accuracy which can be obtained will depend upon the accuracy of the tuning fork. In order to obtain an accuracy of onehundredth of I per cent, the tuning fork must be so arranged that it will vibrate freely and with the same amplitude as was used 
during calibration. Also, the rate of the fork must be corrected for any changes in temperature. Its mounting must be the same as that used during calibration.

In measuring intervals of one-tenth of a second with an accuracy of one-tenth per cent, the recorded interval can be read directly from the film without the use of a comparator. If a freely vibrating tuning fork is used, no error will be introduced on its account. Also, greater flexibility is permissible in the recording mechanism, although the types of apparatus which can be used are still quite limited, since they must not introduce an error of as much as one ten-thousandth of a second.

\section{INTERVALS OF ONE SECOND.}

In order to record time intervals of the order of one second on a plain cylindrical drum, it is necessary to use a comparatively slow speed of film, since it is not feasible to use drums having a circumference of more than $1 / 2$ or $2 \mathrm{~m}$. If the record extends the full length of the film, the accuracy that can be obtained is relatively the same as in the case of the tenth-second interval given above. For these intervals it is desirable to use a 50-cycle tuning fork. Such a fork can be self-driven, which is an advantage if the required accuracy is not too high.

In order to use a high-film speed on a relatively long-time interval, it is necessary to use either a long film running from one drum to another or a wide film with a spiraling device. The latter method has been developed by Doctors Eckhardt and Karcher, of this bureau.

\section{EXPERIMENTAL DETERMINATION OF ACCURACY.}

The accuracy which can be obtained in measuring time intervals of different lengths can be experimentally determined by recording the same interval on two different oscillographs. This has frequently been done, and the results show that with shorttime intervals, where tuning-fork errors are negligible, the difference between the two measurements rarely exceeds $x / 200$ of the timing-line interval. This assumes sharp timing lines about $\mathrm{I} \mathrm{cm}$ apart. If a 500-cycle fork is used, this means that the uncertainty in the time interval is about five-millionths of a second.

With longer time intervals, which require a meter or more of film, slight variations in the frequencies of the forks make it 
impossible to determine the errors due to other causes. To determine the magnitude of the errors on a long film, due to other causes than the fork, a set-up was arranged, so that one tuning fork gave the timing lines for two different oscillographs. Measurements made in this way show that the absolute error due to film shrinkage, acceleration of film, and inaccuracies in reading the film are the same for long intervals as for short intervals. The results of measurements on one pair of films are given in Table 2.

TABLE 2.-Accuracy of Time Determinations of Films.

\begin{tabular}{|c|c|}
\hline $\begin{array}{c}\text { Time length } \\
\text { measured. }\end{array}$ & $\begin{array}{c}\text { Difference in time } \\
\text { over two fims. }\end{array}$ \\
\hline $\begin{array}{c}\text { Miiliseconds. } \\
6.3 \\
33.5\end{array}$ & Millisecond. \\
120.6 & 0.008 \\
& .007 \\
\hline
\end{tabular}

The results of these measurements show that the difference is of the same order of magnitude for all time intervals measured, the maximum being eight microseconds.

\section{SUMMARY.}

The errors which arise in measuring short-time intervals by means of a tuning fork, which throws flashes of light on a photographic film, are discussed. If the conditions are such that the errors in the tuning fork are negligible, then the error is of the order of five-millionths of a second regardless of the length of interval measured. A tuning fork will easily give an accuracy of o.I per cent, but an accuracy of o.or per cent can only be attained by giving careful consideration of the factors which affect the period, such as temperature, amplitude, mounting, and method of drive. Hence, for intervals less than five milliseconds the errors due to the fork are generally less than those due to the recording apparatus, but for longer intervals the fork errors are likely to predominate.

WASHINGTON, November 24, 1922. 